\title{
Enhancing PAPR reduction for FBMC-OQAM systems by joint both tone reservation and companding methods
}

\author{
Salima Senhadji ${ }^{1}$, Yassine Mohammed Bendimerad ${ }^{2}$, Fathi Tarik Bendimerad $^{3}$ \\ ${ }^{1,3}$ LTT Laboratory, Department of Telecommunication, University Abou Baker Belkaid, Algeria \\ ${ }^{2}$ Department of Electrical and Electronic Engineering, University of Bechar, Algeria
}

\begin{tabular}{l} 
Article Info \\
\hline Article history: \\
Received Jun 15, 2020 \\
Revised Aug 11, 2020 \\
Accepted Aug 21, 2020 \\
\hline Keywords: \\
FBMC-OQAM \\
Mu-law companding \\
PAPR reduction \\
TR \\
TR\&Compd
\end{tabular}

\section{Corresponding Author:}

Salima Senhadji

LTT Laboratory

Department of Telecommunication

Abou Baker Belkaid University

Tlemcen, 1300, Algeria

Email: salima.senhadji@student.univ-tlemcen.dz

\begin{abstract}
As all multi-carrier designs FBMC-OQAM signal suffers from large peak-toaverage power ratio (PAPR). There are divers PAPR reduction methods to solve this problem. Tone reservation (TR) scheme is one of the most famous PAPR reduction techniques in which a peak cancelling signal is added to the original one in such a way that PAPR will reduce. Companding is another easy PAPR reduction technique in which compression of large amplitude samples and expansion of low one. In this paper, we suggest a new PAPR reduction scheme called TR\&Compd, based on combining tone reservation and Mu-law companding techniques for FBMC-OQAM systems. The simulation results show that the new proposed scheme (TR\&Compd) presents better results in term of PAPR reduction compared to TR and Mulaw companding methods taken separately.
\end{abstract}

This is an open access article under the CC BY-SA license.

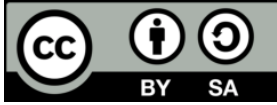

\section{INTRODUCTION}

The multicarrier modulation technique (MCM) is widely used in wireless communication systems such as LTE, 4G, Wimax and also for the next generation 5G. MCM presents a lot of advantages; the most interesting is (i) the robustness against multipath propagation and (ii) narrowband interference. Till now, Orthogonal Frequency Division Multiplex (OFDM) is the most important class of MCM systems. However, it is a limited version of the new $5 \mathrm{G}$ vision due to some drawbacks.

The Filter Bank Multi-Carrier (FBMC) based systems combined with Offset Quadrature Amplitude Modulation (OQAM) [1-5] are gaining appeal to be the favorite to become the radio waveform in forthcoming fifth Generation (5G) physical layer [6]. This advanced modulation scheme offers lots of advantages such as excellent frequency localization [7, 8], low side lobes in its PSD (Power Spectral Density), robustness to phase noise and frequency offsets making it more suitable than OFDM for 5G [9]. Nevertheless, as all multicarrier designs, FBMC suffers from large Peak-to Average Power Ratio (PAPR) which leads to saturation of the high power amplifier (HPA) and cause some in band and out of band distortions which impact the signal quality.

Divers PAPR reduction schemes have been suggested. The most used techniques are: Clipping and Filtering [10], Coding schemes [11], Nonlinear Companding Transforms [12, 13], tone reservation (TR) [14, 15], Selected Mapping (SLM) [16, 17], and Partial Transmit Sequences (PTS) [18]. In the literature, there 
have been many works focusing on the PAPR reduction in FBMC-OQAM systems. In [19], a hybrid solution with conventional SLM and TR was proposed to achieve better PAPR reduction. also in [20], a solution called TR-PTS hybrid scheme with a multi data block-PTS by taking advantage of the FBMC-OQAM symbol overlaps was proposed to enhance PAPR reduction. Other works use companding technique only as in [21], the PAPR of the FBMC system is reduced directly by using A-law and $\mu$-law companding functions at the expense of a certain BER performance.

In this paper, we propose a new hybrid scheme called TR\&Compd for PAPR reduction in FBMCOQAM signals which joint tone reservation (TR) and Mu-Law companding methods. For the new TR\&Compd scheme, the first step is to further process the original signals through the TR scheme. Since some peaks power of the FBMC-OQAM signals usually occur at a few points, we propose to perform MuLaw companding function in order to enhance PAPR reduction performance. The simulation results are used to show the effectiveness of the proposed TR\&Compd scheme in reducing the power envelope fluctuations for FBMC-OQAM signals.

The rest of the paper is organized as follows: Section 2, include a description of FBMC-OQAM system, the PAPR problem and HPA model, also we present a background about the tone reservation technique of PAPR reduction, Mu-law companding and the proposed algorithm (TR\&Compd). Section 3, shows the simulation results and analysis. Finally, we end the paper with some conclusions in Section 4.

\section{RESEARCH METHODS}

\subsection{FBMC-OQAM system and PAPR problem}

The input of the Filter Bank Multi-Carrier with Offset Quadrature Amplitude Modulation (FBMC-

OQAM system) [22] is generally a complex symbol, which is defined as follows:

$$
S_{m}^{n}=\mathfrak{R}_{m}^{n}+j \mathfrak{I}_{m}^{n}
$$

Where $m \in[0, M-1]$ and $n \in[0, N-1], \mathfrak{R}_{m}^{n}$ and $\mathfrak{I}_{m}^{n}$, denote the real and imaginary parts of the $m^{\text {th }}$ complex symbol $S_{m}^{n}$ on the $n^{\text {th }}$ subcarrier respectively. The number of the subcarriers is $N$ and the complex input symbols numbers is. The FBMC-OQAM system transmitter side is shown in Figure 1.

For OQAM, the real and imaginary parts of the complex symbols QAM are staggered in time domain by $T / 2$, where $T$ is the symbol period (equivalent to $N$ ). Then, the symbols are passed through a filter bank of transmission. The $m^{\text {th }}$ transmission FBMC-OQAM symbol can be obtained by adding all subcarriers, which can be expressed as follow:

$$
S^{\prime \prime}(t)=\sum_{n=0}^{N-1}\left[\Re_{m}^{n} \lambda(t-m T)+j \Im_{m}^{n} \lambda\left(t-m T-\frac{T}{2}\right)\right] e^{j n\left(\frac{2 \pi}{T} t+\frac{\pi}{2}\right)}
$$

Where $\lambda(t)$ is the prototype filter impulse response.

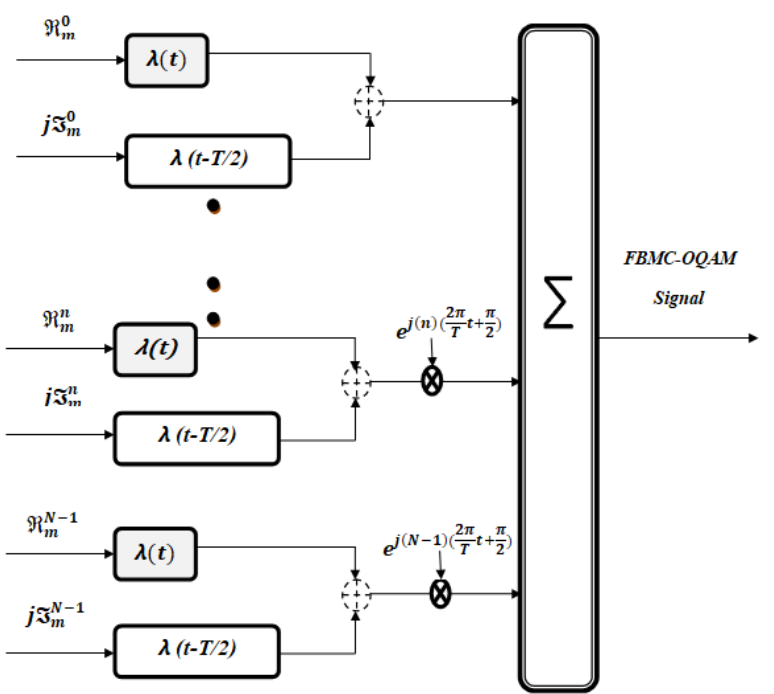

Figure 1. The FBMC-OQAM transmitter 
In this paper, PHYDYAS [23] filter is considered as the prototype filter. It is characterized by an overlapping factor noted $\rho$ and the design of this filter is based on the frequency sampling technology. The impulse response of the PHYDYAS filter is given as [23] in (3) followed by the frequency domain coefficient of the filter as shown in Table 1.

$$
\lambda(t)=\left\{\begin{array}{c}
\frac{1}{\sqrt{B}}\left[1+2 \sum_{k=1}^{\rho-1}(-1)^{k} G_{k} \cos \left(\frac{2 \pi k t}{\rho T}\right)\right], t \in[0, \rho T] \\
0 \text { elsewher }
\end{array}\right.
$$

Where:

$$
\left\{\begin{array}{c}
B=\rho T\left[1+2 \sum_{k=1}^{\rho-1} G_{k}^{2}\right] \\
G_{k}=0, k>3 \\
G_{k}=G_{-k}, k<0
\end{array}\right.
$$

And

Table 1. The frequency domain coefficient of the PHYDYAS filter

\begin{tabular}{cccc}
\hline$G_{0}$ & $G_{1}$ & $G_{2}$ & $G_{3}$ \\
\hline 1 & 0.9716960 & $\frac{1}{\sqrt{2}}$ & $\sqrt{1-G_{1}^{2}=0.235147}$
\end{tabular}

After filtering, the FBMC-OQAM symbol duration is extended by $\rho T$, which means that $\rho-1$ consecutive output symbols overlap with each other in the time domain. After the summation of the $M$ FBMC-OQAM symbols the length is $(M+\rho-1 / 2) T$ where $S(t)$ is given as:

$$
S(t)=\sum_{m=0}^{M-1} S_{m}^{\prime \prime}(t), t \in\left[0,\left(M+\rho-\frac{1}{2}\right) T\right]
$$

For PAPR analysis, in this paper we consider only the middle area of the FBMC-OQAM signals from $(\rho-1 / 2) N / 2$ to $M N+(\rho-1 / 2) N / 2$ with $M N$ points that is then divided into $M$ intervals equally with time duration $T$. Thus, the PAPR of each interval is defined by (6) and, we use the complementary cumulative distribution function (CCDF) presented by (7) to plot it.

$$
P A P R(d B)=10 \log _{10} \frac{\max _{i t \leq t \leq(i+1) T}\left(|s(t)|^{2}\right)}{E\left(|s(t)|^{2}\right)}
$$

Where $E\{\cdot\}$ denotes the expected value and $i \in[0, M]$.

$$
C C D F(P A P R)=\operatorname{Pr}\left(P A P R>P A P R_{0}\right)=1-\left(1-e^{-\Delta}\right)^{N}
$$

A high power amplifier (HPA) is one of the indispensable components in the wireless mobile communication systems. In this paper, we consider a HPA follows the Rapp model with a smoothness factor $\alpha$ that control the transition between the linear zone and the saturation one of HPA.The Rapp model [24] presents only AM/AM conversion. This one can be expressed as:

$$
\begin{aligned}
& F_{A M / A M}(s(t))=\frac{s(t)}{\left(1+\left(\frac{s(t)}{A_{s a t}}\right)^{2 \alpha}\right)^{\frac{1}{2 \alpha}}} \\
& F_{A M / P M}(s(t))=0
\end{aligned}
$$

Where the smoothness factor is all time $\alpha>0$.

\subsection{Tone reservation merhod for FBMC-OQAM PAPR reduction}

TR is a linear technique was first introduced in [14], but requires an efficient generation of the peakcanceling signal. The PAPR can be reduced by using the Tone Reservation (TR) technique which exploits a

\footnotetext{
Enhancing PAPR reduction for FBMC-OQAM systems by joint both tone reservation... (Salima Senhadji)
} 
small number of unused subcarriers (reserved tones) to generate a peak-canceling signal [15]. In TR scheme based on iterative clipping filtering algorithm for FBMC-OQAM systems. The total $N$ subcarriers (tones) are partitioned into $P$ peak reduction tones (PRTs) and $N-P$ data tones. The positions of the PRTs are known by the receiver and transmitter. The input complex symbol in frequency domain can be written as:

$$
\begin{aligned}
& S_{m}^{n}=F_{m}^{n}+C_{m}^{n}=\left\{\begin{array}{l}
C_{m}^{n}, n \in P \\
F_{m}^{n}, n \in P^{C}
\end{array}\right. \\
& C_{m}^{n}=0, \text { for } n \in P^{C} \text { and } F_{m}^{n}=0, \text { for } n \in P
\end{aligned}
$$

Where $S_{m}^{n}$ is $m^{\text {th }}$ data block composed of the data vector $F_{m}^{n}$ and $C_{m}^{n}$ the PAPR reduction vector. $P=$ $\left\{n_{1}, n_{2}, \ldots . n_{P}\right\}$ denotes the set of tones reserved for peak reduction, $P^{C}$ denotes the set of data subcarriers, and $P^{C}$ is the complement set of $P$ in $\mathbb{N}=\{1,2, \ldots \ldots N\}$. The data tones in time domain $\Gamma(t)$ are generated by $N$ point IFFT operation of $Z_{m}^{n}$. The main steps are as:

First step: $\Gamma(t)$ is clipped to a threshold $\beta$ as:

$$
\widetilde{\Gamma(t)}=\left\{\begin{array}{c}
\Gamma(t),|\Gamma(t)| \leq \beta \\
\beta e^{j \varphi_{i}},|\Gamma(t)|>\beta
\end{array}\right.
$$

Where $\Gamma(t)=|\Gamma(t)| e^{j \varphi_{i}}, \varphi_{i}$ is the phase of $\Gamma(t)$.

Second step: the original clipping noise $z(t)$ is calculated as:

$$
\begin{aligned}
& z(t)=\widetilde{\Gamma(t)}-\Gamma(t) \\
& C_{m}^{n}=\left\{\begin{array}{l}
Z, n \in P \\
0, n \in P^{C}
\end{array}\right.
\end{aligned}
$$

Where $Z=F F T(z)$.

Third step: $c(t)$ is added to $s(t)$ and PAPR of the peak reduced FBMC-OQAM signal can be expressed as:

$$
P A P R_{T R}(d B)=10 \log _{10} \frac{\max _{i T \leq t \leq(i+1) T}|s(t)+c(t)|^{2}}{E\left[|s(t)|^{2}\right]}
$$

Where $c(t)$ is time domain peak reduction signal.

\subsection{Mu-law companding method}

Companding is a PAPR reduction technique considered as amplitude limiting scheme, easy to implement in any numerical system. Different types of nonlinear companding are discussed in literature as Alaw, Mu-law notted also ( $\mu$-law) companding. In companding technique, using different functions the higher amplitudes are compressed and smaller amplitudes are expanded resulting in reduction of PAPR. For the given input signal $s$, the mathematical formula of the output signal of Mu-law companding and decompanding techniques are $D$ and $\bar{D}$ respectively given as:

$$
\begin{aligned}
& D=\frac{V \log \left(1+\frac{\mu|s|}{V}\right)}{\log (1+\mu)} \operatorname{sgn}(s)(15) \\
& \bar{D}=\frac{V}{\mu}\left(e^{\frac{|y| \log (1+\mu)}{V}}-1\right)(16)
\end{aligned}
$$

Where $\mu$ is the Mu-law parameter of the compander, $s$ is input signal, and $V$ is the peak amplitude of signal $s$ [25]. $\log$ is the natural logarithm, and $\operatorname{sgn}$ is the signum function. $y$ is the received signal at the receiver.

\subsection{Proposed joint TR\&Compd scheme for FBMC-OQAM PAPR reduction}

In this subsection, we present our proposed scheme (TR\&Compd) which combines the TR scheme and the Mu-law companding method to enhance the PAPR reduction in FBMC-OQAM systems. These two schemes can be complementary, the FBMC-OQAM signal goes through the TR PAPR reduction block in 
order to reduce its high envelope fluctuations, but a few peaks of the complex symbol cannot be canceled by this way only, and will be affected by the non linearity of the power amplifier. For this, we propose to apply Mu-law companding function to enhance high peaks reduction. This proposed PAPR reduction approach combines the advantages of linearity from the first step (TR) with the reduced computation complexity of the second step (Mu-law), providing a better PAPR reduction with good efficiency. The steps of the proposed TR\&Compd scheme are described as below:

Proposed algorithm

1. Sepecify a desired threshold $\beta$, reserved tones $P$ and the maximal iteration numbers $I$ for TR scheme.

2. Generate the time domain signal $s(t)$ of all the $M$ data blocks at the output of the FBMC-OQAM transmitter using (6).

3. Clip the FBMC-OQAM signal at $\beta$ by using (11).

4. Generate a peak cancelling signal by :

- Calculate the clipping noise using (12) $z(t)$.

- Convert it to the frequency domain $Z(f)$.

- Remodulate $C(f)$ to get $c(t)$.

5. Calculate the new TR-FBMC-OQAM signal by adding $c(t)$ to $s(t)$.

6. Applay Mu-law Companding to the TR-FBMC-OQAM signal i.e., $w(t)=$ $D[c(t)+s(t)]$ using (15).

7. Calculate the PAPR of the new TR\&Compd FBMC-OQAM signal by (7).

\section{RESULTS AND DISCUSSION}

In this section, to estimate the performance of the proposed scheme TR\&Compd for FBMC-OQAM system PAPR reduction we have done extensive simulations. Mu-law compander is used because theoretically it gives better BER compared to A-law compander. Table 2 shows the simulation parameters used in this paper for FBMC-OQAM system, Tone Reservation, Mu-law companding and HPA Rapp model. From the CCDFs curves (Figure 2 and the numerical values reported in Table 3), we can see that the PAPR of the unchanged FBMC-OQAM signal is $10 \mathrm{~dB}$, For a $C C D F=10^{-3}$. Moreover, when the CCDF is $10^{-3}$, among three schemes (TR, Compd, TR\&Compd), TR\&Compd scheme decreases by a gain equal to $3.7 d B$, TR decreases by $2.8 \mathrm{~dB}$, while Compd decreases the PAPR by $1.8 \mathrm{~dB}$. It can be observed that the proposed TR\&Compd performs better than TR and Companding separately in terms of PAPR reduction.

Table 2. The simuation parameters

\begin{tabular}{ll}
\hline Parameters & Values \\
\hline Modulation model & 4 OQAM \\
Overlapping factor & $\rho=4$ \\
Number of data blocks & $M=16$ \\
Number of subcarriers & $N=64$ \\
Number of PRTs & $P=8$ \\
Number of iteration for TR & $I=8$ \\
Threshold & $\beta=2$ \\
Mu-ratio & $\mu=1$ \\
Smoothness factor & $\alpha=3$ \\
IBO & $3-6 d B$ \\
\hline
\end{tabular}

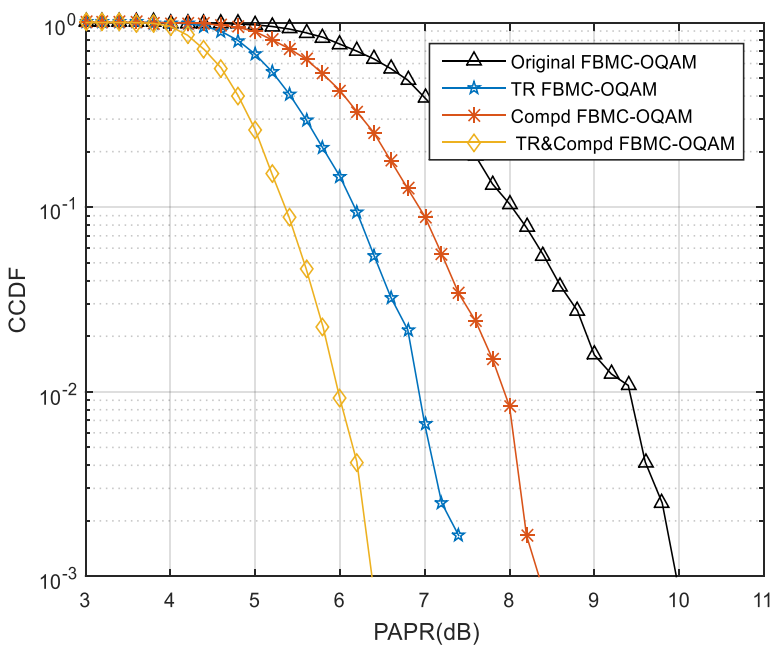

Figure 2. CCDFs of TR, Compd, TR\&Compd schemes and original FBMC-OQAM signal

Table 3. The CCDF values at $10^{-3}$

\begin{tabular}{ccccc}
\hline Algorithms & Original & TR & Companding & TR\&Compd \\
\hline CCDF Values & $10 \mathrm{~dB}$ & $7.3 \mathrm{~dB}$ & $8.2 \mathrm{~dB}$ & $6.2 \mathrm{~dB}$ \\
\hline
\end{tabular}

Enhancing PAPR reduction for FBMC-OQAM systems by joint both tone reservation... (Salima Senhadji) 
We have performed the simulation (see Figure 3. (a)) with different Mu-ratio $\mu=1,2,4$ when the fixed parameters are $\beta=2, P=8, I=8$. From Figure 3. (a), we note that the PAPR decreases as the increasing of Mu-ratio. For example, when $\mu=1$ and $C C D F=10^{-3}$, the PAPR value of the proposed scheme is $6.5 d B$. For $\mu=2,4$ respectively, at $C C D F=10^{-3}$, the PAPR is $6.1 d B$ and $5.1 d B$. It can be verified that the suggested scheme have improved the peak reduction (PAPR). Then, different number of iterations $I=4,8$ and 16 with the fixed parameters $\mu=1, P=8, I=8$ are considered in Figure 3 . (b). We note that the gain of PAPR reduction increases by increasing the iterations number, for $I=16$ the gain of reduction is $4.2 \mathrm{~dB}$. In Figure 3. (c), we show the PAPR threshold of the proposed scheme using different clipping thresholds $\beta$ with fixed parameters: $P=8, I=8, \mu=1 . \quad \beta$ is selected as $\beta=1.6,2,2.2$ respectively. It is illustrated that the small threshold $(\beta)$ may not contribute in reducing PAPR for the suggested way. For example, when $C C D F=10^{-3}$, the PAPR values of the combined scheme with $\beta=$ 1.6, 2 and 2.2 are about $6.55 d B, 6.57 d B$ and $6.35 d B$, respectively. As simulation results show, it can be verified that the proposed TR\&Compd scheme have improved the PAPR reduction in FBMC/OQAM system.

Figure 4. (a) and (b) compare the BER performances of TR scheme, TR \&Compd and FBMCOQAM signals in the presence of HPA Rapp model for $I B O=3 d B$ and $I B O=6 d B$ and $\alpha=3$ over Additive White Gaussian Noise (AWGN) channel. From both figures, we can notice that the BER performance of TR\&Compd is slightly degraded than TR scheme only. We can deal this degradation by adding a simple precoding to greatly improve the overall system performance.

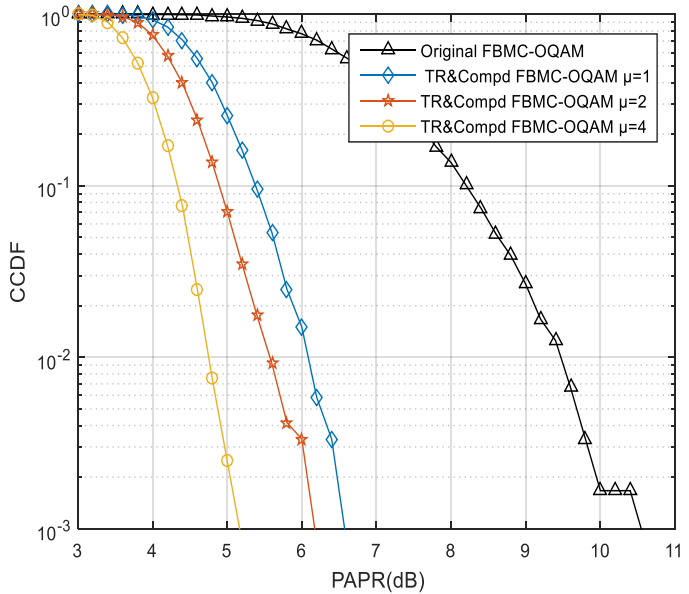

(a)

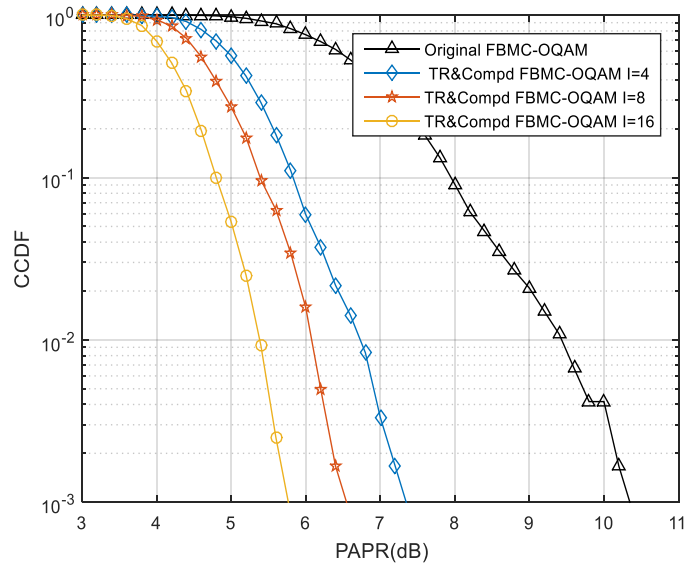

(b)

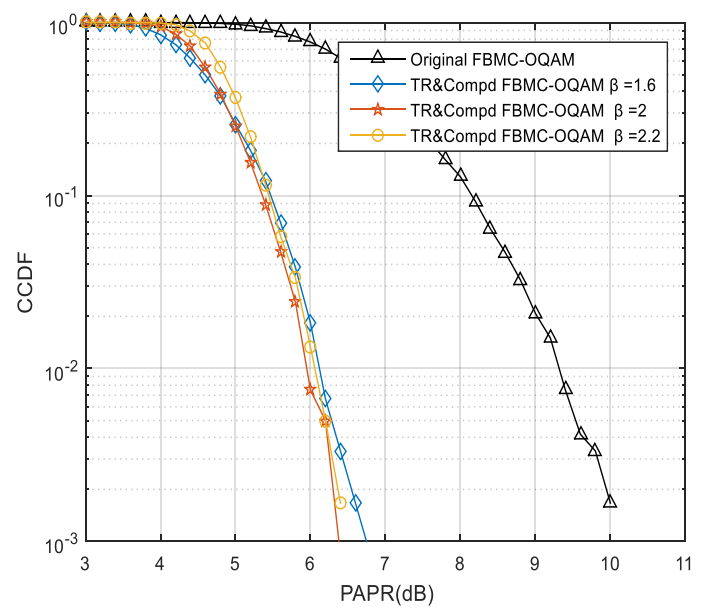

(c)

Figure 3. CCDFs of TR\&Compd scheme with different parameters 


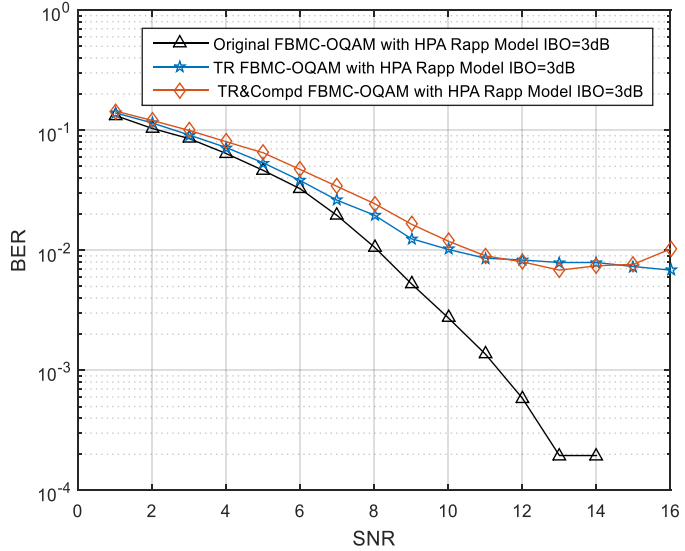

(a)

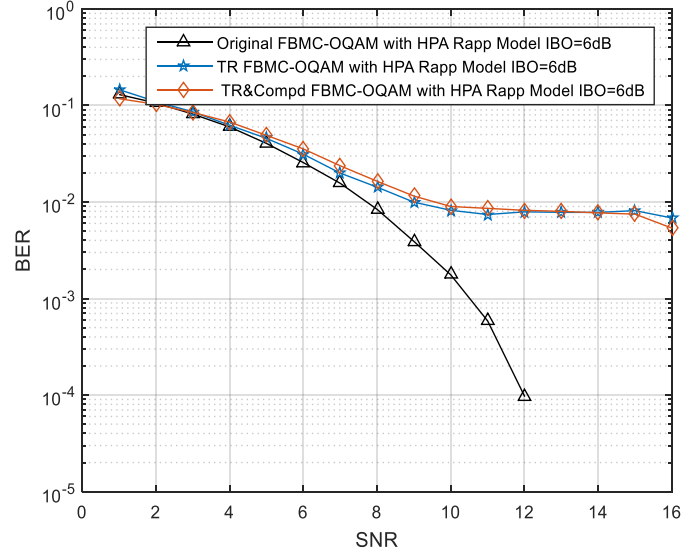

(b)

Figure 4. BER performance of TR, TR\&Compd schemes and Original FBMC-OQAM in presence of HPA Rapp model over AWGN channel for $\mathrm{IBO}=3 \mathrm{~dB}(\mathrm{a}), \mathrm{IBO}=6 \mathrm{~dB}$ (b)

\section{CONCLUSION}

In this paper, we proposed a combined scheme TR\&Compd based on both Tone Reservation approach and the Companding (Mu-law) technique. The proposed approach can reduce the PAPR on FBMCOQAM signals in a significant way. Based on the present findings, this proposed serial approach outperforms the separate use of TR and Companding schemes in terms of PAPR gain reduction. Moreover, the serial combination in terms of BER in the presence of a HPA with amplitude distortion it is noted that the BER performance slightly degrades with using the joint schemes but we can deal this degradation by using any sort of precoding.

\section{ACKNOWLEDGEMENTS}

This work was supported by the national project with technological development and socioeconomic impact under code $\mathrm{N}^{\circ} 13-2019-D G R S D T /$ Univ Tlemcen, entitled: contribution to the spectral and energetic efficiency of $5 \mathrm{G}$ communications systems.

\section{REFERENCES}

[1] FP7-ICT PHYDYAS Project, Prototype Filter and Structure Optimization, Project Deliverable D5.1, (Section 5.2.3), accessed on Jan. 22, 2009. [Online]. Available: http://www.ict-phydyas.org/delivrables

[2] T. Ihalainen, et al., "Filter bank based multi-mode multiple access scheme for wireless uplink," in Proc Eur Signal Process Conf, pp. 1354-1358, 2009,.

[3] R. Youssef, et al., "Distributed coding/modulation scheme with OFDM/OQAM based transmission for wireless cooperative relays systems, " in Proc 24th IEEE Int Symp Pers Indoor Mobile Radio Commun, pp. 1196-1200, 2013.

[4] T. Maksymyuk and V. Pelishok, "The LTE channel transmission rate increasing," in Proc Int Conf Modern Problems Radio Eng Telecommun Comput Sci, pp. 2512-52, 2012.

[5] J. Nadal, et al., "Low-complexity pipelined architecture for FBMC/OQAM transmitter, " in IEEET rans Circuits Syst II Express Briefs, vol. 63, no. 1, pp. 19-23, 2016.

[6] P. Mogensen et al., "5G small cell optimized radio design, International Workshop on Emerging Technologies for LTE-Advanced and Beyond 4G, " in 2013 IEEE Globecom Workshops, Atlanta, GA, pp. 111-116, 2013.

[7] T.Li, et al., "Powers pectral density comparison for the clipped OFDM-types ignals, " in Proc $2 n$ dInt Conf Netw. Secur Wireless Commun Trusted Comput, pp. 269-272, 2010.

[8] R. Razavi, et al., "Information theoretic analysis of OFDM/OQAM with utilized intrinsic interference, " IEEE Signal Process Lett, vol. 22, no. 5, pp. 618-622, 2015.

[9] B. Farhang-Boroujeny, "OFDM Versus Filter Bank Multi-carrier, " IEEE Signal Processing Magazine, vol. 8, no. 3, pp. 92-112, 2006.

[10] Y.C. Wang and Z.Q. Luo, "Optimized iterative clipping and filtering for PAPR reduction of OFDM signals," IEEE Trans.Commun, vol.59, no.1, pp. 33-37, 2011.

[11] E. Jones, et al., "Block coding scheme for reduction of peak to mean envelope power ratio of multicarrier transmission schemes," in IEEE Electronics Letters, vol. 30, no. 8, pp. 2098-2099, 1994. 
[12] X. B. Wang, et al., "Reduction of peak-to-average power ratio of OFDM system using a companding technique," IEEE Trans Broadcasting, vol. 45, no. 3, pp. 303-307, 1999.

[13] T. Jiang, et al., "Exponential companding transform for PAPR reduction in OFDM systems," IEEE Trans. Broadcasting, vol. 51, no. 2, pp. 244-248, 2005.

[14] J. Tellado, and J. Cioffi, "Peak power reduction for multicarrier transmission," in IEEE CTMC GLOBECOM, Sydney, Australia, 1998.

[15] J. Tellado, "Peak to average power ratio reduction for multicarrier modulation," PhD thesis, University of Stanford, Stanford, 1999.

[16] S. S. Yoo, et al., "A novel PAPR reduction scheme for OFDM systems: Selective mapping of partial tones (SMOPT), " in IEEE Trans. Consumer Electronics, vol. 52, no. 1, pp. 40-43, 2006.

[17] R. W. Bauml, et al., "Reducing the peak-to-average power ratio of multicarrier modulation by selected mapping, " iIEEE Electronics Letters, vol. 32, no. 22, pp. 2056-2057, 1996.

[18] S. H. Muller and J. B. Huber, "OFDM with reduced peak-to-average power ratio by optimum combination of partial transmit sequences," in IEEE Electronics Letters, vol. 33, no. 5, pp. 368-369, 1997.

[19] S. Vangala, S. A nuradha, "Hybrid PAPR reduction scheme with selective mapping and tone reservation for FBMC/OQAM," in Proc 3rd International Signal Processing Communication and Networking, Chennai, India, pp. $1-5,2015$.

[20] H. Wang, et al., "Hybrid PAPR reduction scheme for FBMC/OQAM systems based on multi data block PTS and TR methods, " in IEEE Access, vol. 4, pp. 4761-4768, 2016.

[21] A. Hasan, et al., "PAPR reduction of FBMC-OQAM using A-law and Mulaw companding," 2018 ELEKTRO, Mikulov, pp. 1-4, 2018.

[22] P. Siohan, et al., "Analysis and design of OFDM/OQAM systems based on filter bank theory," in IEEE Trans. Signal Process, vol. 50, no. 5, pp. 1170-1183.

[23] M. Bellanger, et al., "FBMC physical layer : A primer, " PHYDYAS, vol. 5, no. 4, pp. 7-10, 2010.

[24] R. Christoph., "Effects of HPA-nonlinearity on a 4-DPSK/OFDM-signal for a digital sound broadcasting signal," In ESA Second European Conference on Satellite Communications (ECSC-2), pp. 179-184, 1991.

[25] Sklar, Bernard, Digital Communications: Fundamentals and Applications, Englewood Cliffs, NJ, Prentice-Hall, 1988. 